\title{
Comparison of Atrial Wave Extraction Methods from Invasive Recordings in Atrial Fibrillation
}

\author{
JJ Rieta ${ }^{1}$, F Hornero ${ }^{2}$, R Alcaraz ${ }^{3}$, D Morata $^{1}$ \\ ${ }^{1}$ Biomedical Synergy. Valencia University of Technology, Spain \\ ${ }^{2}$ Cardiac Surgery Department. General University Hospital of Valencia Foundation, Spain \\ ${ }^{3}$ Innovation in Bioengineering. Castilla-La Mancha University, Spain
}

\begin{abstract}
A key issue to understand the pathophysiological mechanisms of atrial fibrillation $(A F)$ is the analysis and interpretation of atrial electrograms (AEG). To properly study these signals, ventricular artifacts have to be removed. This work presents a new application of independent component analysis (ICA) to reduce ventricular artifacts from AEG recordings making use of only one reference lead. Therefore the technique is suitable when multi-lead recordings are unavailable as in atrial implantable cardioverterdefibrilators. The methodology has been compared with traditional techniques on a database of 20 patients. Performance was evaluated through atrial waveform similarity and ventricular activity reduction as a function of atrial rhythm regularity on a beat-by-beat basis. When the atrial activity is quite regular, results show that ICA preserves the atrial waveform better than the other methods whereas maintaining ventricular reduction.
\end{abstract}

\section{Introduction}

Atrial Fibrillation (AF) is a very common cardiovascular disease in clinical practice affecting up to $1 \%$ of the general population [1]. Considering its prevalence with age, this arrhythmia affects up to $15 \%$ of the population older than 70 and has an incidence that doubles with each advancing decade [1]. One relevant issue to understand its mechanisms is the analysis and interpretation of atrial electrograms (AEG), which are recordings obtained on the atrial surface. To properly study AF signals, ventricular artifacts have to be removed from the AEG. In the context of invasive recordings, some well-known methods are based on template matching and subtraction (TMS), where direct suppression of the QRS complex and $\mathrm{T}$ wave is performed [2]. Other recently presented alternative, initially proposed for ECG recordings, consist of applying signal separation algorithms, that can be based on principal component analysis (PCA) [3] or independent compo- nent analysis (ICA) [4]. ICA has been applied to classify different atrial tachyarrhythmias from multichannel electrograms [5] and to remove recording artifacts (different from ventricular activity) in multichannel atrial mapping techniques [6]. However, multichannel AEG recordings are not always available (for example on AICD environments), thus causing to, initially, discard ICA-based techniques for these situations.

In the present contribution, a new application of ICA is presented where ventricular artifacts from AEGs are removed making use of only one reference lead. The methodology is compared both with the well-known TMS technique and with adaptive cancellation. For the three methodologies, a performance study was carried out as a function of atrial rhythm regularity. The study proves that each approach can behave differently depending on the atrial signal regularity.

\section{Materials}

Twenty patients undergoing cardiac surgery that developed postoperative AF were selected. Ten of the selected patients were in type I AF, characterized by a high degree of regularity in the atrial waveform, and the remaining patients were in type III AF, with notably irregular atrial waveform. The selection of AF types was provided by expert cardiologists following Wells classification [7].

For each patient, surface and epicardial leads were obtained simultaneously. Epicardial electrodes were placed on the right atrium free wall after the surgery procedure. Next, one segment of 30 seconds in length was selected. The sampling frequency in all cases was $1 \mathrm{kHz}$. The selected segments from each recording were notch filtered $\left(f_{n}=50 \mathrm{~Hz}\right)$ to cancel out powerline interference, highpass filtered $\left(f_{h}=0.5 \mathrm{~Hz}\right)$ to suppress base line wandering and low-pass filtered $\left(f_{l}=70 \mathrm{~Hz}\right)$ to reduce high frequency noise [8]. All the filtering operations were performed bidirectionally to avoid phase distortion. 


\section{Ventricular cancellation techniques}

The TMS methodology takes advantage of the lack of a fixed relationship between atrial and ventricular activities and the consistent morphology of the QRST complexes [2]. In this method, an average beat is generated and replicated to create a template that can be subtracted from the original signal, resulting in the remainder AEG with subtracted ventricular activity.

Adaptive Ventricular Cancellation (AVC) is based on adaptive filtering [9]. In our case the main channel, $m(t)=a(t)+v_{a}(t)$, was the recorded AEG containing both atrial and ventricular components, where $a(t)$ is the atrial activity and $v_{a}(t)$ is the ventricular artifact on the AEG. The reference channel, $v_{r}(t)$, was lead II from the standard surface ECG. This lead was selected because of the large ventricular amplitude that can be observed on it. The error signal or cost function is the difference between the desired and the estimated signals, therefore

$$
e(t)=a(t)+v_{a}(t)-\sum_{k=0}^{P-1} w(k) v_{r}(t-k),
$$

which is the output of the whole system and is also used to adjust the coefficients $w(k)$ of the adaptive filter by minimizing the mean square error of $e(t)$. Bearing in mind that the reference ventricular channel $v_{r}(t)$ is clearly correlated with the ventricular artifact $v_{a}(t)$ on the main channel, the error signal will be basically composed of the atrial activity part $a(t)$ from the main channel. After considering different alternatives, the FIR filter number of coefficients was set to $P=10$ and a natural gradient LMS approach was used for the convergence algorithm [9].

Finally, ICA has been considered because in the context of AF patients, atrial and ventricular activities can be considered as decoupled electrical processes [4]. Therefore, it should be possible to dissociate atrial from ventricular activity in one AEG lead by using the proper reference signal which, in this case, will be the surface standard lead II by the same reasons as with AVC. ICA consists of recovering a set of source signals from the observation of linear mixtures of the sources. Nothing is known about the source signals or the mixing structure, the only hypothesis being the source mutual independence [10]. Mathematically, let us denote $\mathbf{s}(t)$ the vector that represents the $N$ source signals, and $\mathbf{x}(t)$ the observation vector, the ICA model for instantaneous linear mixtures reads

$$
\mathbf{x}(t)=\mathbf{A} \mathbf{s}(t)
$$

where $\mathbf{A}$ is the unknown mixing matrix. The objective of ICA is to estimate $\mathbf{s}(t)$ and $\mathbf{A}$ from the exclusive knowledge of $\mathbf{x}(t)$. The FastICA algorithm was preferred to perform the ICA process due to its fast convergence and robust performance [10].

\section{Performance evaluation}

Performance was evaluated by dividing the AEGs into atrial and ventricular segments. A ventricular segment was defined as a $150 \mathrm{~ms}$ time interval centered on the R-peak. Atrial segments were composed of the remaining parts. Within the ventricular segments, performance was evaluated by estimating the ventricular depolarization reduction $(V D R)$, i.e., the beat-by-beat reduction of the R-peak amplitude achieved by the algorithm under evaluation. Therefore, the $V D R$ was a vector of values defined as

$$
V D R(\mathrm{~dB})=10 \log \left(\mathrm{R}_{\mathrm{AEG}} / \mathrm{R}_{\mathrm{VR}}\right)
$$

where $R_{A E G}$ is the R-peak amplitude of the original AEG, and $R_{V R}$ is the residual R-peak amplitude of the atrial electrogram after ventricular activity reduction. High positive values of $V D R$ will indicate good performance of the algorithm. Values close to zero are associated with poor performance and negative values indicate reduction errors, because the peak is larger than before.

For atrial segments, performance was evaluated by measuring the waveform similarity $(S)$, which was evaluated by estimating the cross correlation coefficient, for each atrial segment, between the original AEG and the ventricular reduced resultant. Thereafter, similarity was a vector of values defined as

$$
S=C_{A E G, V R} /\left(\sigma_{A E G} \sigma_{V R}\right)
$$

where $C_{A E G, V R}$ is the covariance of the two atrial segments under evaluation (original and ventricular reduced), and $\sigma_{A E G}$ and $\sigma_{V R}$ are their standard deviations, respectively. The similarity will provide information about how ventricular reduction algorithms preserve the atrial waveform in those intervals (atrial intervals) where atrial activity should remain unchanged.

\section{Results and discussion}

In Fig. 1 and Fig. 2 examples of both type I and type III AF recordings are shown. Only five seconds are included for clarity. Observe in Fig. 1(a) how TMS can modify the atrial waveform within the atrial segments, whereas AVC and ICA may present lower ventricular reduction. Fig. 1(b) plots the resulting similarity index $(S)$ and Fig. 1(c) shows the $V D R$ index. Fig. 2(a) plots results for a type III AF recording. In this case, TMS performs better preserving the atrial waveform and reducing ventricular peaks. Fig. 2(b) shows similarity where a notable performance decrease of AVC and ICA can be observed.

All the recordings in the database were analyzed in a similar way. The simultaneous statistical comparison Kruskal-Wallis test for type I AF gave $p=0$ for $S$ and $p=0.002$ for $V D R$, respectively. Regarding type III 

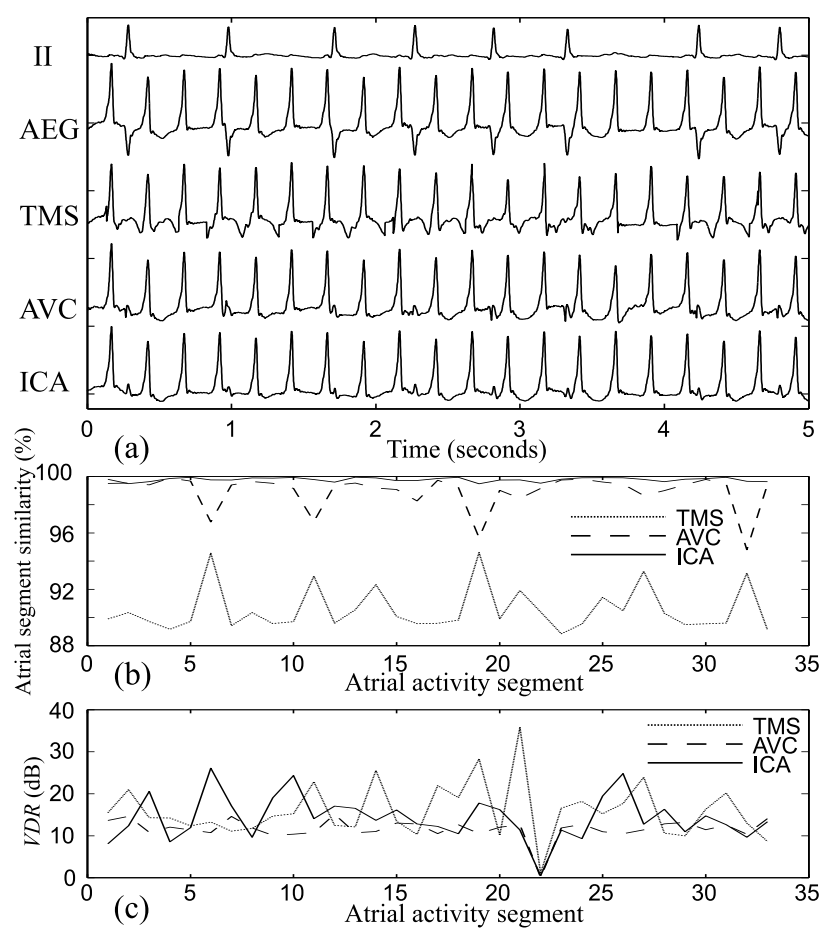

Figure 1. (a) From top to bottom, Lead II of type I AF, AEG and result of ventricular reduction for TMS, AVC and ICA. (b) Similarity $S$ between the atrial segments beatby-beat. (c) Ventricular depolarization reduction $(V D R)$ between the AEG-R peaks and the reduced atrial signal.

Table 1. $S$ and $V D R$ for the two AF types. Values indicate Median \pm Median absolute deviation.

\begin{tabular}{|c|c|c|c|c|c|}
\hline type & $\mathrm{S}(\%)$ & $\operatorname{VDR}(\mathrm{dB})$ & type I & $\mathrm{S}(\%)$ & $\operatorname{VDR}(\mathrm{dB})$ \\
\hline$\overline{\mathrm{TMS}}$ & $95.18 \pm 2.71$ & $4.98 \pm 4.48$ & $\overline{\mathrm{TMS}}$ & $97.72 \pm 1.87$ & $7.91 \pm 3.23$ \\
\hline $\mathrm{AVC}$ & $94.76 \pm 4.12$ & $4.12 \pm 2.72$ & $\mathrm{AVC}$ & $93.74 \pm 4.38$ & $10.46 \pm 3.22$ \\
\hline ICA & $99.64 \pm 0.31$ & $6.32 \pm 4.41$ & ICA & $98.22 \pm 1.53$ & $7.01 \pm 3.25$ \\
\hline
\end{tabular}

$\mathrm{AF}$, result was $p=0$ both for $S$ and $V D R$. Thus, results were statistically distinguishable. Fig. 3 shows a box and whisker plot for $S$. Due to the significant number of outliers in the data, median values (Md) and median absolute deviation (MAD) were computed (see Table 1 for numerical details). As can be seen, ICA offers the best performance, but also presents some outliers. TMS and AVC seem to behave similarly, though TMS is more stable on its results (lower MAD). Regarding type III AF, ICA still is the best method for waveform preservation, but present larger outliers. TMS performance is very close to ICA, being AVC the method that gives the poorer results.

Finally, results for VDR are summarized in Fig. 4 and Table 1. For type I AF patients, ICA present the best results, whereas TMS and AVC present quite similar results. Regarding type III AF, AVC is the methodology that best behaves, giving TMS and ICA similar results.
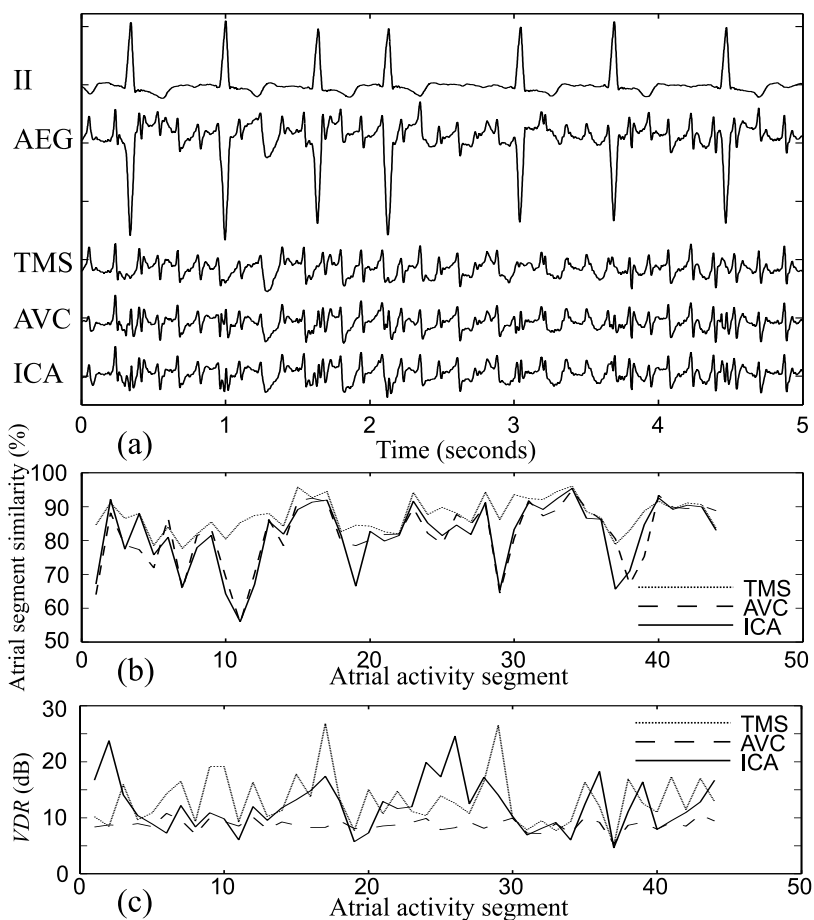

Figure 2. (a) From top to bottom, Lead II of type III AF, AEG and result of ventricular reduction for TMS, AVC and ICA. (b) Similarity $S$ between the atrial segments beatby-beat. (c) Ventricular depolarization reduction $(V D R)$ between the AEG-R peaks and the reduced atrial signal.

The differentiation between type I and III AF patients has shown performance differences. When $\mathrm{AF}$ is quite organized, ICA preserves the atrial waveform notably better than the other methods. In the case of TMS, the average ventricular activity template can be repetitively influenced by the atrial wave. This provokes atrial signal deformation and lowers the similarity. On the other hand, when the atrial activity is disorganized, TMS notably improves performance whereas AVC and ICA decrease in waveform preservation.

The behaviour of AVC and ICA is strongly dependent on an adequate reference. Baseline fluctuations outside the QRS complex and R-peak time misalignments will decrease performance. The large number of outliers for all the $S$ and $V D R$ cases (Figs. 3 and 4) indicates that the three methods can fail independently on the AF type.

\section{Conclusions}

Three alternatives for ventricular activity reduction in atrial electrograms have been presented. Considering that the most widely extended methodology is TMS, this contribution has demonstrated its reasonable performance with respect to other alternatives when the atrial activity 


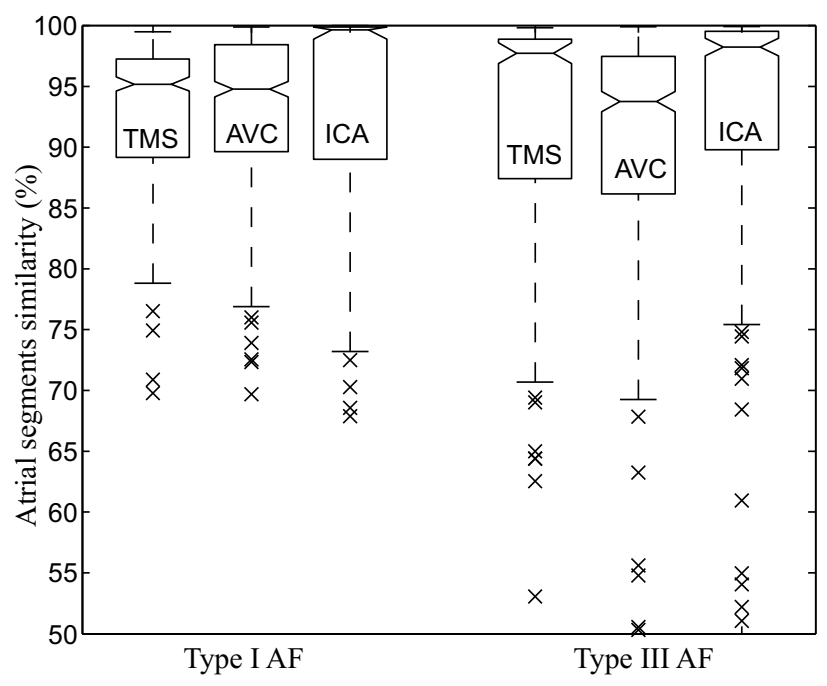

Figure 3. Atrial segments similarity between original and ventricular-reduced atrial electrograms. Boxes on the left are Type I AF patients and on the right are Type III.

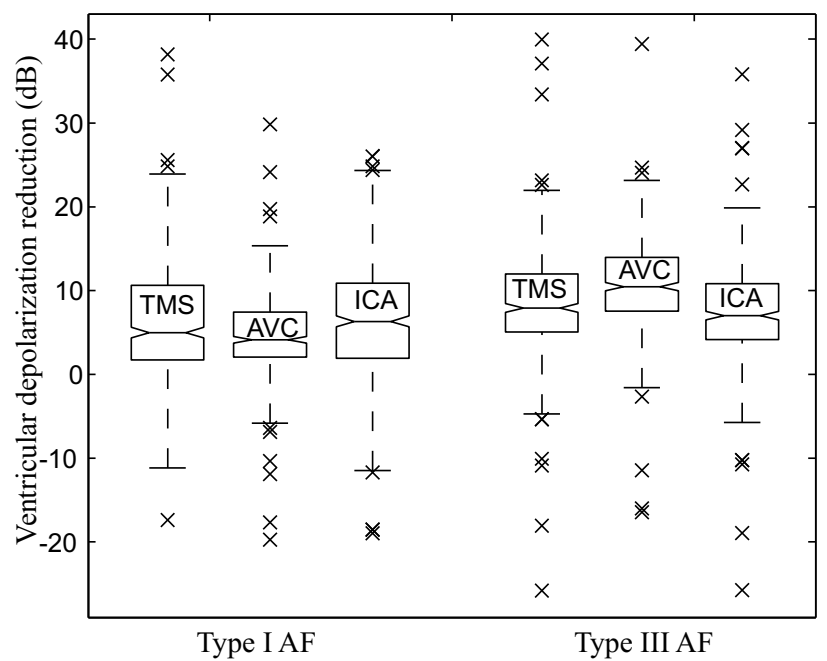

Figure 4. Box plots of ventricular depolarization reduction for the three methodologies. Boxes on the left are Type I AF patients and on the right are Type III AF patients.

is notably disorganized. On the other hand, for organized atrial activity, TMS can produce atrial signal deformation and, then, ICA is the recommended alternative because atrial waveform is very precisely maintained and ventricular artifacts are satisfactory reduced.

Epicardial atrial waves are a relevant tool in the study and management of AF. This information can be provided by AICD or via other invasive techniques where multi-lead recordings are unavailable. Within this context, methods to reduce ventricular artifacts and to preserve atrial wave are crucial for later atrial activity analysis.

\section{Acknowledgements}

This work was partly supported by the projects 20070086 from the R+D+i Vice-rectorate of the Valencia University of Technology, GV06/299 from Consellería de Empresa, Universidad y Ciencia de la Generalitat Valenciana and TEC2007-64884 from the Spanish Ministry of Education and Science.

\section{References}

[1] Mehra R, Ziegler P, Sarkar S, Ritscher D, Warman E. Management of atrial tachyarrhythmias. rhythm control using implantable devices. IEEE Engineering in Medicine and Biology Magazine 2006;25(6):52-62.

[2] Petrutiu S, Nijm GM, Angari HA, Swiryn S, Sahakian AV. Atrial fibrillation and waveform characterization - A time domain perspective in the surface ECG. IEEE Engineering in Medicine and Biology Magazine 2006;25(6):24-30.

[3] Raine D, Langley P, Murray A, Furniss SS, Bourke JP. Surface atrial frequency analysis in patients with atrial fibrillation: Assessing the effects of linear left atrial ablation. Journal of Cardiovascular Electrophysiology 2005;16(8):838844.

[4] Rieta JJ, Castells F, Snchez C, Zarzoso V, Millet J. Atrial activity extraction for atrial fibrillation analysis using blind source separation. IEEE Trans Biomed Eng 2004; 51(7):1176-1186.

[5] Govindan A, Deng G, Kalman J, Power J. Independent component analysis applied to electrogram classification during atrial fibrillation. In Fourteenth International Conference on Pattern Recognition, volume 14. IEEE, 1998;

[6] Liu JH, Kao T. Removing artifacts from atrial epicardial signals during atrial fibrillation. International Conference on Independent Component Analysis and Blind Signal Separation ICA 2003;4:179-183, Nara, Japan.

[7] Wells JL, Karp RB, Kouchoukos NT, MacLean WA, James TN, Waldo AL. Characterization of atrial fibrillation in man: studies following open heart surgery. Pacing and Clinical Electrophysiology PACE 1978;1(4):426-438.

[8] Sörnmo L, Laguna P. Bioelectrical Signal Processing in Cardiac and Neurological Applications. Elsevier Academic Press, 2005. ISBN 0-12-437552-9.

[9] Widrow B, Glover JR, McCool JM, et al. Adaptive noise cancelling: Principles and applications. Proceedings of the IEEE 1975;63(12):1692-1716.

[10] Hyvarinen A, Karhunen J, Oja E. Independent Component Analysis. John Wiley \& Sons, Inc., 2001.

Address for correspondence:

José Joaquín Rieta

Valencia University of Technology

Campus Gandia

46730, GANDIA (Valencia), Spain

E-mail: jjrieta@eln.upv.es 